\title{
On Duality between Local Maximum Stable Sets of a Graph and its Line-Graph
}

\author{
Vadim E. Levit \\ Department of Computer Science and Mathematics \\ Ariel University Center of Samaria, ISRAEL \\ levitv@ariel.ac.il \\ Eugen Mandrescu \\ Department of Computer Science \\ Holon Institute of Technology, ISRAEL \\ eugen_m@hit.ac.il
}

\begin{abstract}
$G$ is a König-Egerváry graph provided $\alpha(G)+\mu(G)=|V(G)|$, where $\mu(G)$ is the size of a maximum matching and $\alpha(G)$ is the cardinality of a maximum stable set, 3], 22.

$S$ is a local maximum stable set of $G$, and we write $S \in \Psi(G)$, if $S$ is a maximum stable set of the subgraph induced by $S \cup N(S)$, where $N(S)$ is the neighborhood of $S$, 12. Nemhauser and Trotter Jr. proved that any $S \in \Psi(G)$ is a subset of a maximum stable set of $G, 20$.

In this paper we demonstrate that if $S \in \Psi(G)$, the subgraph $H$ induced by $S \cup N(S)$ is a König-Egerváry graph, and $M$ is a maximum matching in $H$, then $M$ is a local maximum stable set in the line graph of $G$.

Keywords: Line graph, König-Egerváry graph, maximum matching, local maximum stable set.
\end{abstract}

\section{Introduction}

Throughout this paper $G=(V, E)$ is a simple (i.e., a finite, undirected, loopless and without multiple edges) graph with vertex set $V=V(G)$ and edge set $E=E(G)$. If $X \subset V$, then $G[X]$ is the subgraph of $G$ spanned by $X$. By $G-W$ we mean the subgraph $G[V-W]$, if $W \subset V(G)$. We also denote by $G-F$ the partial subgraph of $G$ obtained by deleting the edges of $F$, for $F \subset E(G)$, and we write shortly $G-e$, whenever $F=\{e\}$. If $A, B \subset V$ are disjoint and non-empty, then by $(A, B)$ we mean the set $\{a b: a b \in E, a \in A, b \in B\}$.

The neighborhood of a vertex $v \in V$ is the set $N(v)=\{w: w \in V$ and $v w \in E\}$. If $|N(v)|=1$, then $v$ is a pendant vertex. We denote the neighborhood of $A \subset V$ by $N_{G}(A)=$ $\{v \in V-A: N(v) \cap A \neq \emptyset\}$ and its closed neighborhood by $N_{G}[A]=A \cup N(A)$, or shortly, $N(A)$ and $N[A]$, if no ambiguity.

$K_{n}, C_{n}$ denote respectively, the complete graph on $n \geq 1$ vertices, and the chordless cycle on $n \geq 3$ vertices. A graph having no $K_{3}$ as a subgraph is a triangle-free graph. 
A stable set in $G$ is a set of pairwise non-adjacent vertices. A stable set of maximum size will be referred to as a maximum stable set of $G$, and the stability number of $G$, denoted by $\alpha(G)$, is the cardinality of a maximum stable set in $G$. In the sequel, by $\Omega(G)$ we denote the set of all maximum stable sets of the graph $G$.

A set $A \subseteq V(G)$ is a local maximum stable set of $G$ if $A$ is a maximum stable set in the subgraph spanned by $N[A]$, i.e., $A \in \Omega(G[N[A]])$, [12. Let $\Psi(G)$ stand for the set of all local maximum stable sets of $G$.

Clearly, every set $S$ consisting of only pendant vertices belongs to $\Psi(G)$. Nevertheless, it is not a must for a local maximum stable set to contain pendant vertices. For instance, $\{e, g\} \in \Psi(G)$, where $G$ is the graph from Figure 1

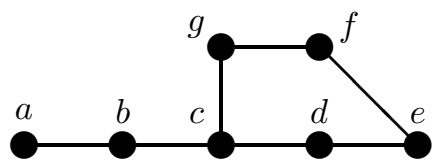

Figure 1: A graph having various local maximum stable sets.

The following theorem concerning maximum stable sets in general graphs, due to Nemhauser and Trotter Jr. [20, shows that some stable sets can be enlarged to maximum stable sets.

Theorem 1.1 [20] Every local maximum stable set of a graph is a subset of a maximum stable set.

Let us notice that the converse of Theorem 1.1 is trivially true, because $\Omega(G) \subseteq \Psi(G)$. The graph $W$ from Figure 1 has the property that any $S \in \Omega(W)$ contains some local maximum stable set, but these local maximum stable sets are of different cardinalities: $\{a, d, f\} \in \Omega(W)$ and $\{a\},\{d, f\} \in \Psi(W)$, while for $\{b, e, g\} \in \Omega(W)$ only $\{e, g\} \in \Psi(W)$.

However, there exists a graph $G$ satisfying $\Psi(G)=\Omega(G)$, e.g., $G=C_{n}$, for $n \geq 4$.

A matching in a graph $G=(V, E)$ is a set of edges $M \subseteq E$ such that no two edges of $M$ share a common vertex. A maximum matching is a matching of maximum size, denoted by $\mu(G)$. A matching is perfect if it saturates all the vertices of the graph. A matching $M=\left\{a_{i} b_{i}: a_{i}, b_{i} \in V(G), 1 \leq i \leq k\right\}$ of a graph $G$ is called a uniquely restricted matching if $M$ is the unique perfect matching of $G\left[\left\{a_{i}, b_{i}: 1 \leq i \leq k\right\}\right]$, [6]. Recently, a generalization of this concept, namely, a subgraph restricted matching has been studied in [5].

Kroghdal found that a matching $M$ of a bipartite graph is uniquely restricted if and only if $M$ is alternating cycle-free (see [10]). This statement was observed for general graphs by Golumbic et al. in [6].

In [12, 13, 16], 17, 18, we showed that, under certain conditions involving uniquely restricted matchings, $\Psi(G)$ forms a greedoid on $V(G)$. The classes of graphs, where greedoids were found include trees, bipartite graphs, triangle-free graphs, and well-covered graphs.

Recall that $G$ is a König-Egerváry graph provided $\alpha(G)+\mu(G)=|V(G)|$ (3], 22]). As a well-known example, any bipartite graph is a König-Egerváry graph (4], [9]). Properties of König-Egerváry graphs were discussed in a number of papers, e.g., 1], 7], 8], 11], 14, [15, 19], 21. Let us notice that if $S$ is a stable set and $M$ is a matching in a graph $G$ such that $|S|+|M|=|V(G)|$, it follows that $S \in \Omega(G), M$ is a maximum matching, and $G$ is a König-Egerváry graph, because $|S|+|M| \leq \alpha(G)+\mu(G) \leq|V(G)|$ is true for any graph. 
The line graph of a graph $G=(V, E)$ is the graph $L(G)=(E, U)$, where $e_{i} e_{j} \in U$ if $e_{i}, e_{j}$ have a common endpoint in $G$.

In this paper we give a sufficient condition in terms of subgraphs of $G$ that ensure that its line graph $L(G)$ has proper local maximum stable sets. In other words, we demonstrate that if: $S \in \Psi(G)$, the subgraph $H$ induced by $S \cup N(S)$ is a König-Egerváry graph, and $M$ is a maximum matching in $H$, then $M$ is a local maximum stable set in the line graph of $G$. It turns out that this is also a sufficient condition for a matching of $G$ to be extendable to a maximum matching.

\section{Maximum matchings and local maximum stable sets}

In a König-Egerváry graph, maximum matchings have a special property, emphasized by the following statement.

Lemma 2.1 [14] Every maximum matching $M$ of a König-Egerváry graph $G$ is contained in each $(S, V(G)-S)$ and $|M|=|V(G)-S|$, where $S \in \Omega(G)$.

For example, $M=\left\{e_{1}, e_{2}, e_{3}\right\}$ is a maximum matching in the König-Egerváry graph $H$ (from Figure 2), $S=\{a, b, c, d\} \in \Omega(H)$ and $M \subset(S, V(H)-S)$. On the other hand, $M_{1}=\{x z, y v\}, M_{2}=\{y z, u v\}$ are maximum matchings in the non-König-Egerváry graph $G$ (depicted in Figure 2), $S=\{x, y\} \in \Omega(G)$ and $M_{1} \subset(S, V(G)-S)$, while $M_{2} \not \subseteq(S, V(G)-S)$.
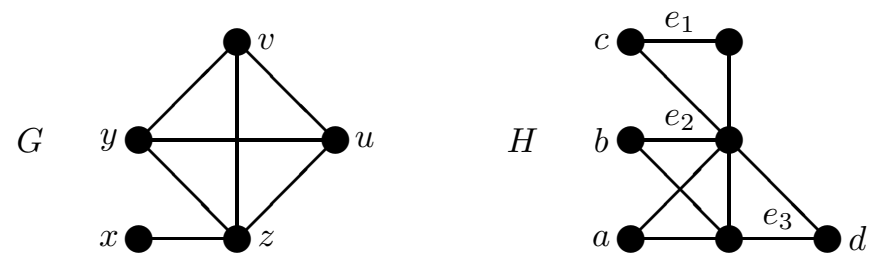

Figure 2: $\{x, y\} \in \Omega(G)$ and $\{a, b, c, d\} \in \Omega(H)$.

Clearly, (maximum) matchings in a graph $G$ correspond to (maximum, respectively) stable sets in $L(G)$ and vice versa. However, not every matching $M$ in $G$ gives birth to a local maximum stable set in $L(G)$, even if $M$ can be enlarged to a maximum matching.
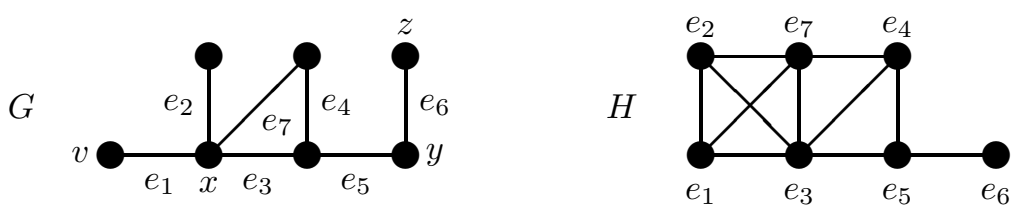

Figure 3: The graph $G$ and its line-graph $H=L(G)$.

For instance, $M_{1}=\left\{e_{1}, e_{6}\right\}, M_{2}=\left\{e_{3}, e_{6}\right\}$ are both matchings in the graph $G$ from Figure 3. but only $M_{1}$ is a local maximum stable set in $L(G)$. Remark that $S_{1}=\{v, z\} \in \Psi(G)$, $S_{2}=\{x, y\} \notin \Psi(G)$ and each $M_{i}$ is a maximum matching in $G\left[N\left[S_{i}\right]\right]$, for $i \in\{1,2\}$. 
Theorem 2.2 If $S \in \Psi(G), H=G[N[S]]$ is a König-Egerváry graph, and $M$ is a maximum matching in $H$, then $M$ is a local maximum stable set in $L(G)$.

Proof. Let $M=\left\{e_{i}=v_{i} w_{i}: 1 \leq i \leq \mu(H)\right\}$. According to Lemma 2.1] it follows that

$$
M \subseteq(S, V(H)-S) \text { and }|M|=|V(H)-S|,
$$

because $H$ is a König-Egerváry graph. Consequently, without loss of generality, we may suppose that

$$
\left\{v_{i}: 1 \leq i \leq \mu(H)\right\} \subseteq S \text {, while } V(H)-S=\left\{w_{i}: 1 \leq i \leq \mu(H)\right\} .
$$

Since $N_{H}\left(v_{i}\right)=N_{G}\left(v_{i}\right) \subseteq N(S)=V(H)-S$, we have that

$$
N_{L(G)}[M]=E(H) \cup\{e=w t \in E: w \in V(H)-S, t \notin S\} .
$$

Hence, every $e \in N_{L(G)}[M]-V(L(H))$ is incident in $G$ to some $w_{i}$.

Assume that $M$ is not a maximum stable set in $L(G)$, i.e., there exists some stable set $Q \subseteq N_{L(G)}[M]$, such that $|Q|>|M|$. In other words, $Q$ is a matching using edges from

$$
E(H) \cup\{e=w t \in E: w \in V(H)-S, t \notin S\},
$$

larger than $M$. Let $F=(M-Q) \cup(Q-M)$. Since $M$ and $Q$ are matchings, every vertex appearing in $G[F]$ has at most one incident edge from each of them, and the maximum degree of a vertex in $G[F]$ is 2 . Hence, $G[F]$ consists of only disjoints chordless paths and cycles. Moreover, every path and every cycle in $G[F]$ alternates between edges of $Q$ and edges of $M$. Since $|Q|>|M|$, it follows that $G[F]$ has a component with more edges of $Q$ than of $M$. Such a component can only be a path, say $P_{x, y}$, that starts and ends with edges from $Q$ (more precisely, from $Q-M$ ) and and $x, y$ are not saturated by edges belonging to $M$. Hence, $P_{x, y}$ must have an odd number of edges.

Case 1. $P_{x, y}$ contains only one edge, namely $x y$. This is not possible, since at least one of the vertices $x, y$ belongs to $V(H)-S$ and is saturated by $M$.

Case 2. $P_{x, y}$ contains at least three edges.

Let $x a, b y \in Q$ be the first and the last edges on $P_{x, y}$. Clearly, $E\left(P_{x, y}\right) \nsubseteq E(H)$, because, otherwise

$$
\left(M-E\left(P_{x, y}\right)\right) \cup\left(E\left(P_{x, y}\right)-M\right)
$$

is a matching in $H$, larger than $M$, in contradiction with the maximality of $M$. Hence, $P_{x, y}$ contains edges from $M$, that alternates with edges from $(E(H)-M) \cup W$, where

$$
W=\{w t \in E(G): w \in V(H)-S, t \in U\},
$$

with

$$
U=\left(S-\left\{v_{i}: 1 \leq i \leq \mu(H)\right\}\right) \cup(V(G)-V(H)\} \neq \varnothing .
$$

Therefore, each second vertex on $P_{x, y}$ must belong to $V(H)-S$. Consequently, we infer that also $y \in V(H)-S$, and hence, it is saturated by $M$, a contradiction.

Notice that $M=\left\{e_{5}, e_{7}\right\} \in \Psi(L(G))$, while there is no $S \in \Psi(G)$, such that $M$ is a maximum matching in $G[N[S]]$, where $G$ is depicted in Figure 4 . In other words, the converse of Theorem 2.2 is not true.

Clearly, every matching can be enlarged to a maximal matching, which is not necessarily a maximum matching. For instance, the graph $G$ in Figure 5 does not contain any maximum matching including the matching $M=\left\{e_{0}, e_{1}, e_{2}\right\}$. The following result shows that, under certain conditions, a matching can be extended to a maximum matching. 

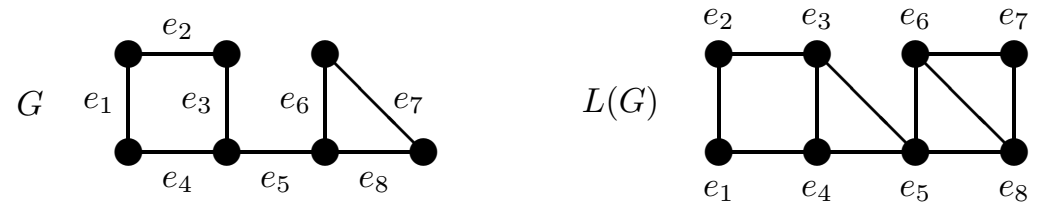

Figure 4: $M=\left\{e_{5}, e_{7}\right\}$ is a matching in $G$ and local maximum stable set in $L(G)$.

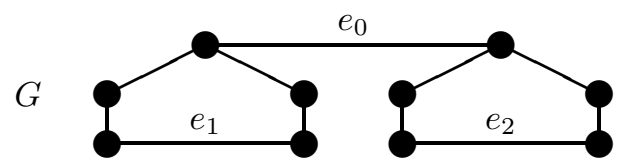

Figure 5: $\left\{e_{0}, e_{1}, e_{2}\right\}$ is a maximal but not a maximum matching.

Corollary 2.3 If $S \in \Psi(G), H=G[N[S]]$ is a König-Egerváry graph, and $M$ is a maximum matching in $H$, then there exists a maximum matching $M_{0}$ in $G$ such that $M \subseteq M_{0}$.

Proof. According to Theorem $2.2 M$ is a local maximum stable set in $L(G)$. By Theorem 1.1. there is some $M_{0} \in \Omega(L(G))$, such that $M \subseteq M_{0}$. Hence, $M_{0}$ is a maximum matching in $G$ containing $M$.

Let us notice that Corollary 2.3 can not be generalized to any subgraph of a non-bipartite König-Egerváry graph.

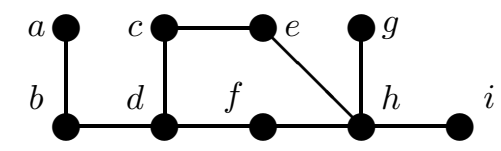

Figure 6: $M=\{a b, c d, f h\}$ is a maximum matching in $N[\{a, c, f\}]$.

For instance, the graph $G$ depicted in Figure [ is a König-Egerváry graph, $S=\{a, c, f\} \in$ $\Psi(G)$, and $M=\{a b, c d, f h\}$ is a maximum matching in $G[N[S]]$, which is not a KönigEgerváry graph, but there is no maximum matching in $G$ that includes $M$.

Since any subgraph of a bipartite graph is also bipartite, we obtain the following result.

Corollary 2.4 If $G$ is a bipartite graph, $S \in \Psi(G)$ and $M$ is a maximum matching in $G[N[S]]$, then there exists a maximum matching $M_{0}$ in $G$ such that $M \subseteq M_{0}$.

\section{Conclusions}

We showed that there is some connection between $\Psi(G)$ and $\Psi(L(G))$.

Let us notice that there are graphs whose line graphs have no proper local maximum stable sets (see the graphs in Figure 7).

Moreover, there are graphs whose iterated line graphs have no proper local maximum stable set, e.g., each $C_{n}$, for $n \geq 3$, since $C_{n}$ and $L\left(C_{n}\right)$ are isomorphic. 

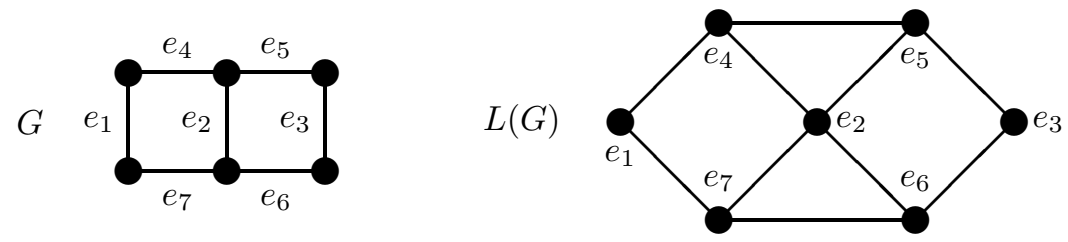

Figure 7: Both $G$ and its line graph $L(G)$ have no local maximum stable sets.

An interesting open question reads as follows. Is it true that for a connected graph $G$ the fact that $L(G)$ has no proper local maximum stable sets implies that $G$ itself does not contain proper local maximum stable sets?

\section{References}

[1] J. M. Bourjolly, W. R. Pulleyblank, König-Egerváry graphs, 2-bicritical graphs and fractional matchings, Discrete Applied Mathematics 24 (1989) 63-82.

[2] G. Chaty, M. Chein, Ordered matchings and matchings without alternating cycles in bipartite graphs, Utilitas Mathematica 16 (1979) 183-187.

[3] R. W. Deming, Independence numbers of graphs - an extension of the König-Egerváry theorem, Discrete Mathematics 27 (1979) 23-33.

[4] E. Egervary, On combinatorial properties of matrices, Mat. Lapok 38 (1931) 16-28.

[5] W. Goddard, S. M. Hedetniemi, S. T. Hedetniemi, R. Laskar, Generalized subgraphrestricted matchings in graphs, Discrete Mathematics 293 (2005) 129-138.

[6] M. C. Golumbic, T. Hirst, M. Lewenstein, Uniquely restricted matchings, Algorithmica 31 (2001) 139-154.

[7] E. Korach, On Dual Integrality, Min-Max Equalities and Algorithms in Combinatorial Programming, University of Waterloo, Department of Combinatorics and Optimization, Ph.D. Thesis, 1982.

[8] E. Korach, T. Nguyen, B. Peis, Subgraph characterization of Red/Blue-Split Graph and König-Egerváry graphs, Proceedings of the Seventeenth Annual ACM-SIAM Symposium on Discrete Algorithms, ACM Press (2006) 842-850.

[9] D. König, Graphen und Matrizen, Mat. Lapok 38 (1931) 116-119.

[10] S. Krogdahl, The dependence graph for bases in matroids, Discrete Mathematics 19 (1977) 47-59.

[11] V. E. Levit, E. Mandrescu, Well-covered and König-Egerváry graphs, Congressus Numerantium 130 (1998) 209-218.

[12] V. E. Levit, E. Mandrescu, A new greedoid: the family of local maximum stable sets of a forest, Discrete Applied Mathematics 124 (2002) 91-101. 
[13] V. E. Levit, E. Mandrescu, Local maximum stable sets in bipartite graphs with uniquely restricted maximum matchings, Discrete Applied Mathematics 132 (2003) 163-174.

[14] V. E. Levit, E. Mandrescu, On $\alpha^{+}$-stable König-Egervary graphs, Discrete Mathematics 263 (2003) 179-190.

[15] V. E. Levit, E. Mandrescu, On $\alpha$-critical edges in König-Egervary graphs, Discrete Mathematics 306 (2006) 1684-1693.

[16] V. E. Levit, E. Mandrescu, Triangle-free graphs with uniquely restricted maximum matchings and their corresponding greedoids, Discrete Applied Mathematics 155 (2007) 2414-2425.

[17] V. E. Levit, E. Mandrescu, Well-covered graphs and greedoids, Theory of Computing 2008. Proceedings of the Fourteenth Computing: The Australasian Theory Symposium (CATS08), Wollongong, NSW. Conferences in Research and Practice in Information Technology, J. Harland and P. Manyem, eds., Volume 77 (2008) 89-94.

[18] V. E. Levit, E. Mandrescu, The clique corona operation and greedoids, Lecture Notes in Computer Science 5165 (2008) 384-392.

[19] L. Lovász, M. D. Plummer, Matching Theory, Annals of Discrete Mathematics 29 (1986) North-Holland.

[20] G. L. Nemhauser, L. E. Trotter, Jr., Vertex packings: structural properties and algorithms, Mathematical Programming 8 (1975) 232-248.

[21] V. T. Paschos, M. Demange, A generalization of König-Egerváry graphs and heuristics for the maximum independent set problem with improved approximation ratios, European Journal of Operational Research 97 (1997) 580-592.

[22] F. Sterboul, A characterization of the graphs in which the transversal number equals the matching number, Journal of Combinatorial Theory Series B 27 (1979) 228-229. 